\title{
2018 UK national guideline for the management of infection with Neisseria
}

gonorrhoeae

Helen Fifer, John Saunders, Suneeta Soni, S Tariq Sadiq, Mark FitzGerald

\section{Changes since 2011 guideline}

- First line empirical treatment is now monotherapy with ceftriaxone $1 \mathrm{~g}$ intramuscularly

- If antimicrobial susceptibility test results from all sites of infection are available prior to treatment and the isolate is sensitive to ciprofloxacin, then this should be used for treatment in preference to ceftriaxone

- Inclusion of testing recommendations in people following genital reconstructive surgery

- Recommendations for extra-genital testing in those with suspected or confirmed antimicrobial resistance

- Epidemiological treatment is recommended only for those presenting within 14 days of exposure. For those presenting after 14 days of exposure we recommend treatment based on the results of testing.

\section{SCOPE AND PURPOSE}

This guideline offers recommendations for the diagnostic tests, treatment regimens and health promotion principles needed for the effective management of gonorrhoea in people aged 16 years and older. For individuals under the age of 16 years please see the BASHH guideline on STI and Related Conditions in Children and Young People. The guidelines are primarily aimed at level 3 sexual health services within the United Kingdom (UK) although the principles of the recommendations could be adopted at all levels.

\section{EDITORIAL INDEPENDENCE}

This guideline was commissioned and edited by the Clinical Effectiveness Group (CEG) of the British Association for Sexual Health and HIV (BASHH), which provided funding for the literature search. No other funding was obtained. 


\section{CONFLICT OF INTEREST}

All authors have signed BASHH conflict of interest forms.

\section{AUTHOR AFFILIATION}

Helen Fifer, Consultant Microbiologist, National Infection Service, Public Health England; John Saunders, Consultant in Sexual Health, National Infection Service, Public Health England and Central and North West London NHS Foundation Trust; Suneeta Soni, Consultant in Sexual Health, Brighton \& Sussex University Hospitals NHS Trust; S Tariq Sadiq, Professor of Molecular Medicine, St George's University of London; Mark FitzGerald, Consultant in Sexual Health; Patient Representative.

\section{RIGOUR OF DEVELOPMENT}

This guideline was produced according to specifications set out in the CEG's 2015 document 'Framework for guideline development and assessment' outlined at https://www.bashh.org/bashh-groups/clinical-effectiveness-group/ and has been updated by reviewing the previous gonorrhoea guideline (2011) and medical literature since its publication. A MEDLINE search of published articles in English language for the years 2009-18 was done using the subject headings 'gonorrhoea' OR 'gonorrhea' OR 'Neisseria gonorrhoeae' AND 'therapy' OR 'treatment' OR 'therapeutics' OR 'resistance' OR 'anti-bacterial agents' OR 'antibiotics' OR 'failure' OR 'toxicity'. All entries in the English language or with abstracts in English were viewed because of the paucity of 'clinical trials' or 'reviews'. The Cochrane Database of Systematic Reviews, Database of Abstracts of Reviews of Effectiveness and Cochrane Controlled Trials Register were reviewed using the textword 'gonorrhoea' and all entries were considered. Abstracts from meetings in the relevant period were hand-searched and considered. Priority was given to randomized controlled trials and systematic review evidence. Recommendations were made and graded on the basis of best available evidence. There is a scarcity of high quality evidence to guide treatment recommendations and therefore, a meeting was held in June 2018 to discuss and resolve differences in opinion surrounding treatment recommendations. This was attended by representatives from the guideline writing group, the CEG, the 
Gonococcal Resistance to Antimicrobials Surveillance Programme (GRASP) Steering Group and the BASHH Bacterial Special Interest Group. Final agreement was achieved by a majority decision following an open vote in this meeting. The draft guideline was appraised with the AGREE instrument, posted on the BASHH website for a consultation period of two months and assessed by a Health Advisor.

\section{PATIENT AND PUBLIC INVOLVEMENT}

A patient representative was a member of the writing group, attended writing group meetings and was involved in the development of the guideline. In addition, attendees for treatment of confirmed gonorrhoea at a specialist sexual health service were consulted on treatment options (including epidemiological treatment) and test of cure.

\section{AETIOLOGY}

Gonorrhoea is caused by the Gram-negative diplococcus Neisseria gonorrhoeae. The primary sites of infection are the columnar epithelium-lined mucous membranes of the urethra, endocervix, rectum, pharynx and conjunctiva. Transmission is by direct inoculation of infected secretions from one mucous membrane to another. Secondary infection to other anatomical sites, through systemic or transluminal spread, can also occur.

\section{CLINICAL FEATURES ${ }^{1-3}$}

Symptoms and signs of infection with gonorrhoea depend, in part, on the site of infection. Co-existing infections and conditions such as Chlamydia trachomatis, Trichomonas vaginalis, Mycoplasma genitalium, Candida albicans and bacterial vaginosis, are not uncommon and these should be considered as a possible cause for an individual's symptoms.

\section{Penile urethral infection}

Symptoms occur in over $90 \%$ of individuals, with discharge and/or dysuria appearing two to five days following exposure. A mucopurulent urethral discharge is often 
present on examination. Rarely, individuals may complain of testicular and epididymal pain with tenderness and swelling present on examination.

\section{Female urethral infection}

Urethral infection may present with dysuria without urinary frequency.

\section{Endocervical infection}

The most common symptom, occurring in about $50 \%$ of individuals, is an increased or altered vaginal discharge. In about a quarter of individuals, lower abdominal pain is present. Gonorrhoea rarely causes intermenstrual bleeding and menorrhagia. On examination, a mucopurulent endocervical discharge may be seen and easily induced endocervical bleeding may be present. However, pelvic and lower abdominal tenderness is an uncommon examination finding in the absence of coinfection with Chlamydia trachomatis.

\section{Rectal infection}

Most cases are asymptomatic but symptoms may include anal discharge and perianal/ anal pain or discomfort. Rectal infection in cisgender women is present in up to a third of cases of urogenital infection, and individuals may not report a history of anal sex. Limited evidence suggests that rectal infection in the absence of urogenital infection is uncommon. ${ }^{4}$

\section{Pharyngeal infection}

This is predominantly asymptomatic but is occasionally associated with a sore throat.

\section{Complicated infection}

Transluminal spread of $N$. gonorrhoeae from the urethra or endocervix may occur and cause epididymo-orchitis, prostatitis or pelvic inflammatory disease (PID). Haematogenous dissemination may occur from infected mucous membranes to cause skin lesions, arthralgia, arthritis and tenosynovitis (disseminated gonococcal infection). In a study involving nearly 4,000 cisgender women attending a sexual 
health clinic in the UK, PID was reported in approximately $14 \%$ of those with gonorrhoea. ${ }^{5}$

\section{DIAGNOSIS AND SPECIMEN COLLECTION}

This section should be read in conjunction with Public Health England's 'Guidance for the detection of gonorrhoea in England', $2014 .{ }^{6}$ The diagnosis of gonorrhoea is established by the detection of $N$. gonorrhoeae at an infected site, either by nucleic acid amplification tests (NAATs) or by culture. The approach and method used to test for gonorrhoea will be influenced by the clinical setting, storage and transport system to the laboratory, local prevalence of infection and the range of tests available in the laboratory. No test for gonorrhoea offers $100 \%$ sensitivity and specificity. ${ }^{6-9}$

\section{Microscopy}

- Microscopy of Gram-stained genital specimens allows direct visualisation of $N$. gonorrhoeae as monomorphic Gram-negative diplococci within polymorphonuclear leukocytes.

- Penile urethra

- Microscopy of urethral or meatal swab smears has good sensitivity (90-95\%) in people with discharge from the penile urethra and is recommended to facilitate immediate presumptive diagnosis in these individuals (Grade 1C). ${ }^{1}$

- Microscopy of penile urethral smears in those without symptoms is less sensitive (50-75\%) therefore, it is not recommended in asymptomatic individuals (Grade 1C). ${ }^{1}$

\section{- Female urethra and endocervix}

- Microscopy has only $37-50 \%$ and $20 \%$ sensitivity compared with culture for detecting gonorrhoea from endocervical and female urethral smears, respectively. ${ }^{3}$

- The sensitivity of cervical microscopy compared to NAATs in a more recent study was only $16 \%{ }^{10}$

- Female urethral and cervical microscopy is therefore not routinely recommended (Grade 1C). 


\section{- Rectum and pharynx}

- Ano-rectal smears and microscopy should be offered if rectal symptoms are present (Grade 1C). ${ }^{11}$

- The sensitivity of microscopy for detecting asymptomatic rectal infection is low and is not recommended (Grade $1 C) .^{12}$

- Microscopy of pharyngeal specimens is not recommended (Grade 1C).

\section{$\underline{\text { Nucleic acid amplification tests }}$}

- NAATs are more sensitive than culture, particularly for oropharyngeal and rectal sites. ${ }^{13-15}$ NAATs show high sensitivity (>95\%) in both symptomatic and asymptomatic infection. ${ }^{14,16,17}$ Therefore, although NAATs are not licensed for use at extra-genital sites, their use is recommended. ${ }^{6}$

- Commercially available NAATs differ in their cross-reactivity to commensal Neisseria species which may be present at significant levels, particularly in the pharynx. ${ }^{18}$ It is recommended that laboratories confirm any reactive test with an alternative molecular target if the positive predictive value of the initial test for the population tested is less than $90 \%$ (Grade 1B). ${ }^{6,7,19}$ This is particularly important for extra-genital specimens.

- Pooling of self-collected or clinician- collected rectal, pharyngeal and urine samples from the same individual could provide cost savings. There is a small evidence base with mixed results using different testing platforms, specimen collection and pooling methods. The largest study to date has shown that pooling of self-taken swabs has lower sensitivity for detection of $N$. gonorrhoeae from pharyngeal sites, when compared with single site testing. ${ }^{20}$ Any service considering the implementation of pooling should perform appropriate clinical evaluation.

\section{- Penile urethra}

- NAATs show equivalent sensitivity in urine and urethral swab specimens from cisgender men although a first-pass urine is the preferred sample. ${ }^{7,21}$ 


\section{- Female urethra and endocervix}

- Self-collected or clinician-collected vulvovaginal swabs perform better than endocervical swabs and significantly better than urine for cisgender women. ${ }^{6}$ , $7,9,22-24$ Vulvovaginal swabs are therefore recommended as the optimal specimen (Grade $1 \mathrm{~A})$.

- For people who have had a hysterectomy, there is no evidence on optimal sampling site. We suggest considering urine and VVS for NAAT with subsequent culture from that site if positive (Grade 2D).

\section{- Rectum and pharynx}

- Infection can occur at multiple sites and an individual can be infected with more than one strain of $N$. gonorrhoeae..$^{25-27}$

- Rectal and pharyngeal sampling should be routine in all men who have sex with men (MSM), as recommended by the BASHH guideline on the sexual health care of MSM, considered in women who are sexual contacts of gonorrhoea and be guided by an assessment of risk and symptoms in everyone else. ${ }^{28-30}$

- Oropharyngeal infection is more difficult to treat. ${ }^{31-34}$ Therefore, anyone with genital gonorrhoea (regardless of gender or reported sexual behaviour) should have pharyngeal sampling if either of the following apply (Grade 1D):

i. Susceptibility results are not available and the infection may have been acquired in the Asia-Pacific region. This is because of high levels of antimicrobial resistance in that region ${ }^{35-38}$ which may lead to treatment failure

ii. Genital infection with a confirmed ceftriaxone resistant strain

\section{Culture}

- The primary role of culture is for antimicrobial susceptibility testing, which is of increasing importance as antimicrobial resistance in $N$. gonorrhoeae continues to evolve and spread. 
- Specimens for culture (urethral, endocervical, neovaginal, anorectal and pharyngeal swabs) should be taken alongside NAATs from people suspected clinically of having gonorrhoea, and from sexual contacts. ${ }^{6}$

- All individuals with gonorrhoea diagnosed by NAAT should have cultures taken for susceptibility testing prior to treatment (Grade 1D).

- For culture, the sensitivity depends on several factors including time from sample collection to plating. Services should seek to minimise this time whether by direct plating in the clinic or use of transport media with prompt transfer for plating in the laboratory.

\section{Considerations for people following genital reconstructive surgery (GRS)}

- The susceptibility of a site to gonococcal infection is likely to be related to the nature of the reconstruction, with sites constructed from mucosal tissue (e.g. from the vaginal or bowel mucosa) being more susceptible than sites constructed from skin.

- Gonococcal infections of the urethra ${ }^{39}$, sigmoid neovagina ${ }^{40}$ and penile skin-lined neovagina ${ }^{41,42}$ have all been reported following GRS. Gonococcal infections of the neopenis are rare.

- The sensitivity of microscopy for the diagnosis of gonococcal infection of the neovagina and neopenis is not known. Examination of a Gram-stained smear from a bowel segment neovagina may facilitate a presumptive diagnosis of gonorrhoea and could be considered (Grade 1D).

- We recommend that optimal genital testing in transgender women at risk of gonorrhoea should include swabs from the neovagina and first-pass urine (Grade 1D).

- We recommend first-pass urine as the specimen of choice from people with a neopenis (Grade 1D). Where the vagina is still present following GRS a vaginal swab should be considered as directed by the sexual history and symptoms.

- Extragenital testing should be guided by sexual history and symptoms.

\section{$\underline{\text { Testing for other STIs }}$}


Approximately $19 \%$ of patients with gonorrhoea have concurrent $C$. trachomatis infection. ${ }^{43}$ Testing for other STIs should be undertaken according to BASHH STI testing guidelines. ${ }^{44}$

\section{Timing of testing}

Infection cannot be ruled out in individuals who test within two weeks of sexual contact with an infected partner. Therefore, it is recommended that patients return for repeat testing after this window period if epidemiological treatment is not given (Grade 1D)..$^{45}$

\section{MANAGEMENT}

\section{General advice}

Patients should be given a detailed explanation of their condition with particular emphasis on the implications for the health of themselves and their partner(s). This should be reinforced, if necessary, with clear and accurate written information (Grade 1D). Patients should be advised to abstain from sexual intercourse until seven days after they and their partner(s) have completed treatment (Grade 1D).

\section{$\underline{\text { Treatment }}$}

\section{Indications for therapy:}

1. Identification of intracellular Gram-negative diplococci on microscopy;

2. A positive culture for N. gonorrhoeae;

3. A confirmed positive NAAT for N. gonorrhoeae;

4. Sexual partner of confirmed case of gonococcal infection (See section below on Sexual Partners);

\section{Treatment of uncomplicated ano-genital and pharyngeal infection in adults}

When antimicrobial susceptibility is not known prior to treatment:

Ceftriaxone $1 \mathrm{~g}$ intramuscularly as a single dose (Grade $1 \mathrm{C}$ )

When antimicrobial susceptibility is known prior to treatment: 
Ciprofloxacin $500 \mathrm{mg}$ orally as a single dose $\mathrm{e}^{46,47}$ (Grade $1 \mathrm{~A}$ ) (See caution in section "Use of ciprofloxacin first line when infection is known to be susceptible")

The prevalence of ciprofloxacin resistance in the UK is high (36.4\% in 2017). ${ }^{43}$ Therefore, we only recommend considering ciprofloxacin as first-line treatment if phenotypic or genotypic antimicrobial susceptibility data indicates susceptibility to ciprofloxacin at all suspected sites of infection. Molecular testing for gyrA gene mutations of NAAT positive gonorrhoea samples is feasible to identify patients who could be treated with ciprofloxacin $27,48,49$ although commercial tests are not currently available in the UK.

The move to ceftriaxone monotherapy represents a major change from the 2011 guideline. There is a lack of high quality evidence regarding the best strategy to delay the emergence of resistance. However, for the reasons outlined below, monotherapy is recommended. A high level of vigilance through use of culture, follow up of patients and test of cure coupled with maintenance of strong surveillance is vital in order to monitor the impact of this approach.

\section{The dose of ceftriaxone has been increased from $500 \mathrm{mg}$ to $1 \mathrm{~g}$}

- The prevalence of ceftriaxone resistance (MIC $>0.125 \mathrm{mg} / \mathrm{L}$ ) is very low in England and Wales, however, there has been an increase in the modal ceftriaxone MIC distribution (i.e. an increase in the proportion of isolates with reduced susceptibility). ${ }^{43,50}$

- Ceftriaxone resistant isolates have been identified in the $\mathrm{UK}^{32,33}$ and globally. ${ }^{51-55}$ The UK cases, which were resistant to both ceftriaxone and azithromycin (including one case of high-level azithromycin resistance, MIC $\geq 256 \mathrm{mg} / \mathrm{L}$ ), had epidemiological links to the Asia-Pacific region where significant levels of reduced susceptibility and resistance to ceftriaxone have been reported. ${ }^{35,36}$

- Although a lower dose of ceftriaxone would be adequate to treat the majority of gonococcal strains that are currently circulating in the UK, data 
suggest that ceftriaxone $1 \mathrm{~g}$ would be more effective against most isolates with increased MICs. ${ }^{56}$

- Therefore, in an attempt to ensure successful treatment of strains with reduced susceptibility, the recommended dose of ceftriaxone has been increased to $1 \mathrm{~g}$.

\section{Dual therapy with azithromycin $1 \mathrm{~g}$ is no longer recommended}

- Azithromycin 1g was added to recommended therapy in 2011 in an attempt to ensure successful treatment of infection with reduced susceptibility to ceftriaxone. ${ }^{56}$ Evidence to suggest synergy between cephalosporins and azithromycin in vitro is inconclusive..$^{57-61}$

- Since 2011 the prevalence of azithromycin resistance in the UK and globally has increased (9.2\% in GRASP 2017, 7.5\% in Euro-GASP 2016). ${ }^{43,62-66}$ There has also been sustained transmission of high-level azithromycin resistant $N$. gonorrhoeae across the $\mathrm{UK}^{67}$ and clusters reported elsewhere. ${ }^{68}$

- Although some of the internationally reported ceftriaxone resistant isolates are susceptible to azithromycin, ${ }^{51,52,55}$ a $1 \mathrm{~g}$ dose of azithromycin may be insufficient to clear infection. In a randomized control trial, the combination of gentamicin $240 \mathrm{mg}$ IM with azithromycin $1 \mathrm{~g}$ only cleared infection in $91 \%$ of participants and did not demonstrate non-inferiority compared to ceftriaxone $500 \mathrm{mg}$ IM with azithromycin $1 \mathrm{~g}$. This suggests a $1 \mathrm{~g}$ dose of azithromycin is insufficient to treat gonorrhoea. ${ }^{69}$

- For infections with azithromycin MICs around the breakpoint $(>0.5 \mathrm{mg} / \mathrm{L})$, a $2 \mathrm{~g}$ dose of azithromycin could potentially be more effective than 1g. However, the $2 \mathrm{~g}$ dose would not be effective against high-level azithromycin resistant isolates. In addition, the incidence of gastrointestinal side-effects is higher with $2 \mathrm{~g}$ azithromycin and so this dose may not be acceptable to patients or clinicians. ${ }^{70}$

- With higher doses of azithromycin, the duration of sub-MIC levels at mucosal surfaces is extended for up to four weeks..$^{71-73}$ If a patient is reinfected with gonorrhoea during this time period, this could potentially select for azithromycin resistance. 
- Other reasons for avoiding azithromycin in the treatment of gonorrhoea centre around antibiotic stewardship in sexual health more generally, particularly the fears of accelerating the induction and spread of resistance in other STIs such as Mycoplasma genitalium and Treponema pallidum.

\section{Use of ciprofloxacin first line when infection is known to be susceptible}

- Using alternative antibiotics where appropriate can reduce the selective pressure which comes from the universal use of ceftriaxone and this may delay the emergence of ceftriaxone resistance. ${ }^{49,74}$

- The writing group are aware of the alert from the European Medicines Agency (EMA) following their 2018 review of serious side effects associated with the use of quinolone and fluoroquinolone antibiotics. ${ }^{75}$ These include side effects involving muscles, tendons, joints and the nervous system. Ciprofloxacin should be avoided in people who have previously had serious side effects with a fluoroquinolone or quinolone antibiotic. It should be used with caution in those over the age of 60 years, those taking a corticosteroid, people with kidney disease and those who have had an organ transplantation.

- Gonorrhoea is a serious infection and the potential benefit of using ciprofloxacin in people with susceptible infection will outweigh the potential risks.

\section{Alternative regimens}

The following options have all been associated with treatment failure when used as monotherapy particularly when used for pharyngeal infection, ${ }^{76-79}$ therefore it is recommended to use dual therapy with azithromycin $2 \mathrm{~g}$ where possible (Grade $2 \mathrm{C}$ ). Clinicians using alternative regimens for empirical treatment of gonorrhoea without antibiotic susceptibility data are recommended to regularly review local and national trends in gonococcal antimicrobial resistance.

Alternative regimens may be given because of allergy, needle phobia or other absolute or relative contraindications. In patients with pencillin allergy there is 
ample evidence to allow the safe use of all but a few early generation cephalosporins (e.g. cephalexin, cefaclor and cefadroxil), and third-generation cephalosporins such as cefixime and ceftriaxone show negligible cross-allergy with penicillins. ${ }^{80,81}$

Therefore, in penicillin-allergic patients ceftriaxone and cefixime are suitable treatment options, unless there is a history of severe hypersensitivity (e.g. anaphylactic reaction) to any beta-lactam antibacterial agent (penicillins, cephalosporins, monobactams and carbapenems). ${ }^{81}$

- Cefixime $400 \mathrm{mg}$ orally as a single dose plus azithromycin $2 \mathrm{~g}$ orally (Grade $1 \mathrm{~B}$ )

- Only advisable if an intramuscular injection is contraindicated or refused by the patient. Resistance to cefixime is currently low in the UK. ${ }^{43}$

- Gentamicin $240 \mathrm{mg}$ intramuscularly as a single dose plus azithromycin $2 \mathrm{~g}$ orally (Grade 1A)

- A large, UK-based, RCT examined the efficacy and safety of gentamicin for the treatment of gonorrhoea. ${ }^{69}$ This study used gentamicin in combination with $1 \mathrm{~g}$ of azithromycin. Microbiological cure (negative NAAT two weeks after treatment) was achieved in $94 \%$ of urogenital, $90 \%$ of rectal and $80 \%$ of pharyngeal infections. Another randomised trial used a $2 \mathrm{~g}$ dose of azithromycin in combination with gentamicin. ${ }^{82}$ This found $100 \%$ clearance of infection however, few extragenital infections were included and culture was used to confirm clearance (i.e. it is likely to overestimate the effectiveness).

- Spectinomycin $2 \mathrm{~g}$ intramuscularly as a single dose plus azithromycin $2 \mathrm{~g}$ orally (Grade 1B)

- Spectinomycin is not recommended for pharyngeal infection because of poor efficacy. ${ }^{31}$ In addition, spectinomycin may be difficult to obtain as it is an unlicensed imported product.

- Azithromycin 2g as a single oral dose (Grade 1B) 
- The clinical efficacy of azithromycin does not always correlate with in vitro susceptibility testing ${ }^{83}$ and azithromycin resistance is high. ${ }^{43}$

\section{Treatment of complicated infections}

\section{Gonococcal PID}

- Ceftriaxone $1 \mathrm{~g}$ intramuscularly as a single dose in addition to the regimen chosen to treat PID (see BASHH PID guideline)

\section{Gonococcal epididymo-orchitis}

- Ceftriaxone $1 \mathrm{~g}$ intramuscularly as a single dose in addition to the regimen chosen to treat epididymo-orchitis (see BASHH epididymo-orchitis guideline)

\section{Gonococcal conjunctivitis}

- Ceftriaxone $1 \mathrm{~g}$ intramuscularly as a single dose (Grade 2D)

- There is a single study of the treatment of gonococcal conjunctivitis conducted in twelve adults. ${ }^{84}$ All were successfully treated with a single dose of ceftriaxone.

- The eye should be irrigated with saline/water.

There is a lack of evidence to guide treatment options if there is a history of penicillin anaphylaxis or established cephalosporin allergy. Treatment should be based on antimicrobial susceptibility results where available.

\section{Disseminated gonococcal infection}

- Ceftriaxone $1 \mathrm{~g}$ intramuscularly or intravenous every 24 hours or

- Cefotaxime $1 \mathrm{~g}$ intravenous every eight hours or

- Ciprofloxacin 500mg intravenous every 12 hours (if the infection is known to be susceptible) or

- Spectinomycin $2 \mathrm{~g}$ intramuscularly every 12 hours 
Therapy should continue for seven days but may be switched $24-48$ hours after symptoms improve to one of the following oral regimens guided by sensitivities:

- Cefixime $400 m g$ twice daily or

- Ciprofloxacin 500mg twice daily or

- Ofloxacin 400mg twice daily

\section{Pregnancy and breastfeeding ${ }^{85-87}$}

Pregnant and breastfeeding individuals should not be treated with quinolone or tetracycline antimicrobials. Pregnancy does not diminish treatment efficacy.

- Ceftriaxone $1 \mathrm{~g}$ intramuscularly as a single dose (Grade $1 \mathrm{~A}$ ) or

- Spectinomycin $2 \mathrm{~g}$ intramuscularly as a single dose (Grade $1 \mathrm{~A}$ )

O Spectinomycin is in the FDA pregnancy category $B$ and therefore not expected to be harmful and can be used if no suitable alternatives. It is not known if it is excreted in breastmilk and should be used with caution in those who are breastfeeding.

- Azithromycin 2g as a single oral dose (Grade 1B)

- The manufacturer of azithromycin advises use only if adequate alternatives are not available. In addition, azithromycin should only be used if isolate known to be susceptible.

\section{HIV-positive individuals}

HIV-positive individuals with gonorrhoea should be managed in the same way as HIV-negative individuals.

\section{Chlamydia coinfection}

- Treatment for confirmed or suspected chlamydia coinfection should follow the current BASHH guideline for the management of chlamydia.

- If an individual has already received azithromycin $2 \mathrm{~g}$ for the treatment of gonorrhoea then this should be sufficient to treat chlamydia and no further doses of azithromycin are required. 


\section{SEXUAL PARTNERS}

\section{Partner notification}

Partner notification should be pursued in all patients identified with gonococcal infection. Action and outcomes should be documented. ${ }^{88}$

Partner notification should follow national recommendations. ${ }^{89}$ The following partners should be notified:

- All partners within the preceding two weeks (or the last partner if longer than two weeks ago) of male patients with symptomatic urethral infection;

- All partners within the preceding three months of patients with infection at other sites or asymptomatic infection.

\section{Treatment of contacts}

Epidemiological treatment is not needed for all sexual contacts, and ideally treatment should only be given to those partners who test positive for gonorrhoea. However, an infection may be missed if a test is performed too soon after a potential exposure. The time between exposure and a positive test result may vary depending on a number of host, pathogen and diagnostic factors. There is a lack of evidence to support recommendations for the optimal time for testing. Therefore, in order to reduce the unnecessary use of antibiotics, we recommend the following as a pragmatic approach:

- For those presenting after 14 days of exposure we recommend treatment only following a positive test for gonorrhoea. ${ }^{90}$

- For those presenting within 14 days of exposure we recommend considering epidemiological treatment based on a clinical risk assessment and following a discussion with the patient. In asymptomatic individuals, it may be appropriate to not give epidemiological treatment, and to repeat testing 2 weeks after exposure.

\section{FOLLOW-UP AND TEST OF CURE (TOC)}


All patients diagnosed with gonorrhoea should be advised to return for TOC with extra emphasis given to patients:

1. With persistent symptoms or signs

2. With pharyngeal infection

3. Treated with anything other than first line recommended regimen when antimicrobial susceptibility unknown

4. Who acquired infection in the Asia-Pacific region when antimicrobial susceptibility unknown

Assessment after treatment may be helpful to:

- detect treatment failure and emerging resistance

- confirm compliance with treatment

- ensure resolution of symptoms

- enquire about adverse reactions

- take a sexual history to explore the possibility of reinfection

- pursue partner notification and health promotion

\section{Method and timing of TOC}

A positive TOC could be due to treatment failure, reinfection or residual non-viable organism and should be interpreted in the clinical context.

- Culture, performed at least 72 hours after completion of therapy, should be used if symptoms or signs are present at time of TOC..$^{91}$

- NAAT should be used if asymptomatic, followed by culture if NAAT-positive.

- The time to a negative TOC using NAATs is variable and there are limited data to inform optimum time to TOC. However, most individuals should be negative seven days following treatment where an RNA NAAT is used and 14 days following treatment when using a DNA NAAT. ${ }^{92}$

- We recommend TOC should be performed at an appropriate time depending on the type of NAAT used (Grade 1B). 


\section{$\underline{\text { Treatment Failures }}$}

Cases of possible ceftriaxone treatment failure in England should be reported to Public Health England using the on-line form:

https://hivstiwebportal.phe.org.uk/login.aspx

Only authorised users are permitted to access this secure website. All specialist sexual health clinics should have access. If required, usernames and passwords can be obtained from gumcad@phe.gov.uk

\section{AUDITABLE OUTCOME MEASURES}

- All individuals with gonorrhoea diagnosed by NAAT should have cultures taken for susceptibility testing prior to treatment (performance standard 97\%)

- Individuals treated for gonorrhoea should have a test of cure performed (performance standard 97\%)

- Individuals diagnosed with gonorrhoea should be tested for all sexually transmitted infections including HIV (unless previously diagnosed with HIV) (performance standard 97\%)

- Individuals diagnosed with gonorrhoea should have partner notification carried out in accordance with the BASHH statement on partner notification (performance standard 97\%)

- Individuals diagnosed with gonorrhoea should be offered information (written or digital) about their diagnosis and management (performance standard 97\%)

- Individuals diagnosed with gonorrhoea should receive first-line treatment or the reasons for not doing so documented (performance standard 97\%)

- Cases of possible treatment failures with ceftriaxone should be reported to Public Health England (performance standard 97\%)

\section{QUALIFYING STATEMENT}

Decisions to follow these recommendations must be based on professional clinical judgement, consideration of individual patient circumstances and available resources. 
All possible care has been undertaken to ensure specification of the correct dosage of medication and route of administration. However, it remains the responsibility of the prescribing clinician to ensure the accuracy and appropriateness of the medication they prescribe.

\section{Time scale for next revision}

An author group will be invited by the BASHH CEG to review and revise the guideline in 2023 using the BASHH framework for guideline development. However, addenda may be issued sooner than 2023, particularly if relevant new data are available relating to treatment or antimicrobial resistance.

\section{Acknowledgements}

We thank the following for their valuable contributions to this guideline: Keith Radcliffe (Chair) and members of the BASHH Clinical Effectiveness Group; Professor Cathy Ison (Chair), Dr Paddy Horner, Dr Michelle Cole and members of the BASHH Bacterial Special Interest Group; Professor David Livermore (Chair), Dr Gwenda Hughes and Professor Jonathan Ross on behalf of the GRASP steering group; Dr Ann Sullivan (Chair), Dr Nisha Pal on behalf of the BASHH National Audit Group; Dr Kate Nambiar on behalf of the BASHH Gender \& Sexual Minorities Special Interest Group; Dr Richard Gilson (University College London); Dr Paul Lee (Medicines and Healthcare products Regulatory Agency); Jane Holder, clinical nurse specialist; patient representative and other patients who gave advice during the development of this guideline; and those who made comments during the web based consultation: Jake Bayley, Rachel Drayton, Anne Edwards, Emily James, David Kellock, Neil Lazaro, Vincent Lee, Nigel O’Farrell, Karin O'Sullivan, Cecilia Priestley, Jonathan Shaw, Jackie Sherrard, Selena Singh, Harriet Wallace, Andrew Whyte, Sathish Thomas William. 


\section{REFERENCES}

1. Sherrard J, Barlow D. Gonorrhoea in men: clinical and diagnostic aspects. Genitourin Med 1996;72(6):422-6.

2. Lewis DA, Bond M, Butt KD, et al. A one-year survey of gonococcal infection seen in the genitourinary medicine department of a London district general hospital. Int J STD AIDS 1999;10(9):588-94. doi: 10.1258/0956462991914717

3. Barlow D, Phillips I. Gonorrhoea in women. Diagnostic, clinical, and laboratory aspects. Lancet 1978;1(8067):761-4.

4. Barry PM, Kent CK, Philip SS, et al. Results of a program to test women for rectal chlamydia and gonorrhea. Obstet Gynecol 2010;115(4):753-9. doi: 10.1097/AOG.0b013e3181d444f6 [published Online First: 2010/03/24]

5. Morris GC, Stewart CM, Schoeman SA, et al. A cross-sectional study showing differences in the clinical diagnosis of pelvic inflammatory disease according to the experience of clinicians: implications for training and audit. Sex Transm Infect 2014;90(6):445-51. doi: 10.1136/sextrans-2014051646

6. Hughes G, Ison C, Field N, et al. Guidance for the detection of gonorrhoea in England. In: Public Health England, ed.

https://assets.publishing.service.gov.uk/government/uploads/system/u ploads/attachment data/file/405293/170215 Gonorrhoea testing guida nce REVISED 2 .pdf, 2014.

7. Whiley DM, Garland SM, Harnett G, et al. Exploring 'best practice' for nucleic acid detection of Neisseria gonorrhoeae. Sex Health 2008;5(1):17-23.

8. Van Dyck E, Ieven M, Pattyn S, et al. Detection of Chlamydia trachomatis and Neisseria gonorrhoeae by enzyme immunoassay, culture, and three nucleic acid amplification tests. J Clin Microbiol 2001;39(5):1751-6. doi: 10.1128/JCM.39.5.1751-1756.2001

9. Cook RL, Hutchison SL, Ostergaard L, et al. Systematic review: noninvasive testing for Chlamydia trachomatis and Neisseria gonorrhoeae. Ann Intern Med 2005;142(11):914-25.

10. Thorley N, Radcliffe K. The performance and clinical utility of cervical microscopy for the diagnosis of gonorrhoea in women in the era of the NAAT. Int J STD AIDS 2015;26(9):656-60. doi: 10.1177/0956462414551233

11. Grover D, Prime KP, Prince MV, et al. Rectal gonorrhoea in men - is microscopy still a useful tool? International Journal of STD \& AIDS 2006;17(4):277-79. doi: 10.1258/095646206776253363

12. Forni J, Miles K, Hamill M. Microscopy detection of rectal gonorrhoea in asymptomatic men. International journal of STD \& AIDS 2009;20(11):7978. doi: 10.1258/ijsa.2009.009186

13. Levy V, Blackmore CS, Klausner JD. Self-collection of specimens for nucleic acid-based diagnosis of pharyngeal, cervicovaginal, urethral, and rectal Neisseria gonorrhoeae and Chlamydia trachomatis infections. Methods Mol Biol 2012;903:407-18. doi: 10.1007/978-1-61779-937-2_28

14. Gaydos CA, Van Der Pol B, Jett-Goheen M, et al. Performance of the Cepheid CT/NG Xpert Rapid PCR Test for Detection of Chlamydia trachomatis and Neisseria gonorrhoeae. J Clin Microbiol 2013;51(6):1666-72. doi: 10.1128/JCM.03461-12 
15. Cornelisse VJ, Chow EP, Huffam S, et al. Increased Detection of Pharyngeal and Rectal Gonorrhea in Men Who Have Sex With Men After Transition From Culture To Nucleic Acid Amplification Testing. Sex Transm Dis 2017;44(2):114-17. doi: 10.1097/OLQ.0000000000000553

16. Van Der Pol B, Hook EW, 3rd, Williams JA, et al. Performance of the BD CTQX and GCQx Amplified Assays on the BD Viper LT Compared With the BD Viper XTR System. Sex Transm Dis 2015;42(9):521-3. doi: 10.1097/OLQ.0000000000000313

17. Geelen TH, Rossen JW, Beerens AM, et al. Performance of cobas(R) 4800 and m2000 real-time assays for detection of Chlamydia trachomatis and Neisseria gonorrhoeae in rectal and self-collected vaginal specimen. Diagn Microbiol Infect Dis 2013;77(2):101-5. doi: 10.1016/j.diagmicrobio.2013.06.020

18. Palmer HM, Mallinson H, Wood RL, et al. Evaluation of the specificities of five DNA amplification methods for the detection of Neisseria gonorrhoeae. $J$ Clin Microbiol 2003;41(2):835-7.

19. Pope CF, Hay P, Alexander S, et al. Positive predictive value of the Becton Dickinson VIPER system and the ProbeTec GC Q x assay, in extracted mode, for detection of Neisseria gonorrhoeae. Sex Transm Infect 2010;86(6):465-9. doi: 10.1136/sti.2010.044065

20. Sultan B, White JA, Fish R, et al. The "3 in 1" Study: Pooling Self-Taken Pharyngeal, Urethral, and Rectal Samples into a Single Sample for Analysis for Detection of Neisseria gonorrhoeae and Chlamydia trachomatis in Men Who Have Sex with Men. J Clin Microbiol 2016;54(3):650-6. doi: 10.1128/JCM.02460-15

21. Chernesky MA, Martin DH, Hook EW, et al. Ability of new APTIMA CT and APTIMA GC assays to detect Chlamydia trachomatis and Neisseria gonorrhoeae in male urine and urethral swabs. J Clin Microbiol 2005;43(1):127-31. doi: 10.1128/JCM.43.1.127-131.2005

22. Schachter J, Chernesky MA, Willis DE, et al. Vaginal swabs are the specimens of choice when screening for Chlamydia trachomatis and Neisseria gonorrhoeae: results from a multicenter evaluation of the APTIMA assays for both infections. Sex Transm Dis 2005;32(12):725-8.

23. Stewart CM, Schoeman SA, Booth RA, et al. Assessment of self taken swabs versus clinician taken swab cultures for diagnosing gonorrhoea in women: single centre, diagnostic accuracy study. BMJ 2012;345:e8107. doi: $10.1136 / \mathrm{bmj} . e 8107$

24. Van Der Pol B, Taylor SN, Liesenfeld O, et al. Vaginal swabs are the optimal specimen for detection of genital Chlamydia trachomatis or Neisseria gonorrhoeae using the Cobas 4800 CT/NG test. Sex Transm Dis 2013;40(3):247-50. doi: 10.1097/OLQ.0b013e3182717833

25. Benn PD, Rooney G, Carder C, et al. Chlamydia trachomatis and Neisseria gonorrhoeae infection and the sexual behaviour of men who have sex with men. Sex Transm Infect 2007;83(2):106-12. doi: 10.1136/sti.2006.021329

26. Lavelle SJ, Jones KE, Mallinson H, et al. Finding, confirming, and managing gonorrhoea in a population screened for chlamydia using the Gen-Probe Aptima Combo2 assay. Sex Transm Infect 2006;82(3):221-4. doi: 10.1136/sti.2005.017616 
27. Pond MJ, Hall CL, Miari VF, et al. Accurate detection of Neisseria gonorrhoeae ciprofloxacin susceptibility directly from genital and extragenital clinical samples: towards genotype-guided antimicrobial therapy. J Antimicrob Chemother 2016;71(4):897-902. doi: 10.1093/jac/dkv432

28. van Liere G, Dukers-Muijrers N, Levels L, et al. High Proportion of Anorectal Chlamydia trachomatis and Neisseria gonorrhoeae After Routine Universal Urogenital and Anorectal Screening in Women Visiting the Sexually Transmitted Infection Clinic. Clin Infect Dis 2017;64(12):170510. doi: $10.1093 / \mathrm{cid} / \mathrm{cix} 243$

29. Jin F, Prestage GP, Kippax SC, et al. Risk factors for genital and anal warts in a prospective cohort of HIV-negative homosexual men: the HIM study. Sex Transm Dis 2007;34(7):488-93. doi: 10.1097/01.olq.0000245960.52668.e5

30. van Liere GA, Hoebe CJ, Niekamp AM, et al. Standard symptom- and sexual history-based testing misses anorectal Chlamydia trachomatis and neisseria gonorrhoeae infections in swingers and men who have sex with men. Sex Transm Dis 2013;40(4):285-9. doi: 10.1097/OLQ.0b013e31828098f8 [published Online First: 2013/03/15]

31. Moran JS, Levine WC. Drugs of choice for the treatment of uncomplicated gonococcal infections. Clin Infect Dis 1995;20 Suppl 1:S47-65.

32. Eyre DW, Sanderson ND, Lord E, et al. Gonorrhoea treatment failure caused by a Neisseria gonorrhoeae strain with combined ceftriaxone and highlevel azithromycin resistance, England, February 2018. Euro Surveill 2018;23(27) doi: 10.2807/1560-7917.ES.2018.23.27.1800323

33. Fifer H, Natarajan U, Jones L, et al. Failure of Dual Antimicrobial Therapy in Treatment of Gonorrhea. N Engl J Med 2016;374(25):2504-6. doi: 10.1056/NEJMc1512757

34. Moran JS. Treating uncomplicated Neisseria gonorrhoeae infections: is the anatomic site of infection important? Sex Transm Dis 1995;22(1):39-47.

35. Chen SC, Yin YP, Dai XQ, et al. First nationwide study regarding ceftriaxone resistance and molecular epidemiology of Neisseria gonorrhoeae in China. J Antimicrob Chemother 2016;71(1):92-9. doi: 10.1093/jac/dkv321

36. Peng T, Lin H, Liu Q, et al. Ceftriaxone susceptibility and molecular characteristics of Neisseria gonorrhoeae isolates in Changsha, China. J Infect Chemother 2017;23(6):385-89. doi: 10.1016/j.jiac.2017.03.007

37. Chen Y, Gong Y, Yang T, et al. Antimicrobial resistance in Neisseria gonorrhoeae in China: a meta-analysis. BMC Infect Dis 2016;16:108. doi: 10.1186/s12879-016-1435-0

38. Jiang FX, Lan Q, Le WJ, et al. Antimicrobial susceptibility of Neisseria gonorrhoeae isolates from Hefei (2014-2015): genetic characteristics of antimicrobial resistance. BMC Infect Dis 2017;17(1):366. doi: 10.1186/s12879-017-2472-z

39. Fiumara NJ, Asvadi S. Asymptomatic gonococcal urethritis in a male transsexual female. Br J Vener Dis 1978;54(2):130-1.

40. van der Sluis WB, Bouman MB, Gijs L, et al. Gonorrhoea of the sigmoid neovagina in a male-to-female transgender. Int J STD AIDS 2015;26(8):595-8. doi: 10.1177/0956462414544725

41. Bodsworth NJ, Price R, Davies SC. Gonococcal infection of the neovagina in a male-to-female transsexual. Sex Transm Dis 1994;21(4):211-2. 
42. Haustein UF. [Pruritus of the artificial vagina of a transsexual patient caused by gonococcal infection]. Hautarzt 1995;46(12):858-9.

43. Public Health England. Key findings from the Gonococcal Resistance to Antimicrobials Surveillance Programme (GRASP) 2017. 2018

44. BASHH Clinical Effectiveness Group. BASHH CEG guidance on tests for Sexually Transmitted Infections. https://www.bashhguidelines.org/media/1084/sti-testing-tables-2015dec-update-4.pdf, 2015.

45. FitzGerald M, Bedford C. National standards for the management of gonorrhoea. Int J STD AIDS 1996;7(4):298-300. doi: 10.1258/0956462961917861

46. Echols RM, Heyd A, O'Keeffe BJ, et al. Single-dose ciprofloxacin for the treatment of uncomplicated gonorrhea: a worldwide summary. Sex Transm Dis 1994;21(6):345-52.

47. Moran JS. Ciprofloxacin for gonorrhea--250 mg or $500 \mathrm{mg}$ ? Sex Transm Dis 1996;23(2):165-7.

48. Allan-Blitz LT, Wang X, Klausner JD. Wild-Type Gyrase A Genotype of Neisseria gonorrhoeae Predicts In Vitro Susceptibility to Ciprofloxacin: A Systematic Review of the Literature and Meta-Analysis. Sex Transm Dis 2017;44(5):261-65. doi: 10.1097/OLQ.0000000000000591

49. Allan-Blitz LT, Humphries RM, Hemarajata P, et al. Implementation of a Rapid Genotypic Assay to Promote Targeted Ciprofloxacin Therapy of Neisseria gonorrhoeae in a Large Health System. Clin Infect Dis 2017;64(9):126870. doi: 10.1093/cid/ciw864 [published Online First: 2016/12/31]

50. Town K, Obi C, Quaye N, et al. Drifting towards ceftriaxone treatment failure in gonorrhoea: risk factor analysis of data from the Gonococcal Resistance to Antimicrobials Surveillance Programme in England and Wales. Sex Transm Infect 2017;93(1):39-45. doi: 10.1136/sextrans-2016-052583

51. Lefebvre B, Martin I, Demczuk W, et al. Ceftriaxone-Resistant Neisseria gonorrhoeae, Canada, 2017. Emerg Infect Dis 2018;24(2) doi: 10.3201/eid2402.171756

52. Camara J, Serra J, Ayats J, et al. Molecular characterization of two high-level ceftriaxone-resistant Neisseria gonorrhoeae isolates detected in Catalonia, Spain. J Antimicrob Chemother 2012;67(8):1858-60. doi: $10.1093 / \mathrm{jac} / \mathrm{dks} 162$

53. Unemo M, Golparian D, Nicholas R, et al. High-level cefixime- and ceftriaxoneresistant Neisseria gonorrhoeae in France: novel penA mosaic allele in a successful international clone causes treatment failure. Antimicrob Agents Chemother 2012;56(3):1273-80. doi: 10.1128/AAC.05760-11

54. Whiley DM, Jennison A, Pearson J, et al. Genetic characterisation of Neisseria gonorrhoeae resistant to both ceftriaxone and azithromycin. Lancet Infect Dis 2018;18(7):717-18. doi: 10.1016/S1473-3099(18)30340-2

55. Lahra MM, Martin I, Demczuk W, et al. Cooperative Recognition of Internationally Disseminated Ceftriaxone-Resistant Neisseria gonorrhoeae Strain. Emerg Infect Dis 2018;24(4) doi: 10.3201/eid2404.171873

56. Chisholm SA, Mouton JW, Lewis DA, et al. Cephalosporin MIC creep among gonococci: time for a pharmacodynamic rethink? J Antimicrob Chemother 2010;65(10):2141-8. doi: 10.1093/jac/dkq289 
57. Barbee LA, Soge 00, Holmes KK, et al. In vitro synergy testing of novel antimicrobial combination therapies against Neisseria gonorrhoeae. $J$ Antimicrob Chemother 2014;69(6):1572-8. doi: 10.1093/jac/dkt540

58. Pereira R, Cole MJ, Ison CA. Combination therapy for gonorrhoea: in vitro synergy testing. J Antimicrob Chemother 2013;68(3):640-3. doi: $10.1093 / \mathrm{jac} / \mathrm{dks} 449$

59. Wind CM, de Vries HJ, van Dam AP. Determination of in vitro synergy for dual antimicrobial therapy against resistant Neisseria gonorrhoeae using Etest and agar dilution. Int J Antimicrob Agents 2015;45(3):305-8. doi: 10.1016/j.ijantimicag.2014.10.020

60. Furuya R, Nakayama H, Kanayama A, et al. In vitro synergistic effects of double combinations of beta-lactams and azithromycin against clinical isolates of Neisseria gonorrhoeae. J Infect Chemother 2006;12(4):172-6. doi: $10.1007 / \mathrm{s} 10156-006-0445-\mathrm{z}$

61. Singh V, Bala M, Bhargava A, et al. In vitro efficacy of 21 dual antimicrobial combinations comprising novel and currently recommended combinations for treatment of drug resistant gonorrhoea in future era. PLoS One 2018;13(3):e0193678. doi: 10.1371/journal.pone.0193678

62. Cole MJ, Spiteri G, Jacobsson S, et al. Overall Low Extended-Spectrum Cephalosporin Resistance but high Azithromycin Resistance in Neisseria gonorrhoeae in 24 European Countries, 2015. BMC Infect Dis 2017;17(1):617. doi: 10.1186/s12879-017-2707-z

63. Yasuda M, Ito S, Hatazaki K, et al. Remarkable increase of Neisseria gonorrhoeae with decreased susceptibility of azithromycin and increase in the failure of azithromycin therapy in male gonococcal urethritis in Sendai in 2015. J Infect Chemother 2016;22(12):841-43. doi: 10.1016/j.jiac.2016.07.012

64. Wi T, Lahra MM, Ndowa F, et al. Antimicrobial resistance in Neisseria gonorrhoeae: Global surveillance and a call for international collaborative action. PLoS Med 2017;14(7):e1002344. doi: 10.1371/journal.pmed.1002344

65. Lahra MM, Ward A, Trembizki E, et al. Treatment guidelines after an outbreak of azithromycin-resistant Neisseria gonorrhoeae in South Australia. Lancet Infect Dis 2017;17(2):133-34. doi: 10.1016/S14733099(17)30007-5

66. Wind CM, Schim van der Loeff MF, van Dam AP, et al. Trends in antimicrobial susceptibility for azithromycin and ceftriaxone in Neisseria gonorrhoeae isolates in Amsterdam, the Netherlands, between 2012 and 2015. Euro Surveill 2017;22(1) doi: 10.2807/1560-7917.ES.2017.22.1.30431

67. Fifer $H$, Cole M, Hughes G, et al. Sustained transmission of high-level azithromycin-resistant Neisseria gonorrhoeae in England: an observational study. Lancet Infect Dis 2018;18(5):573-81. doi: 10.1016/S1473-3099(18)30122-1

68. Katz AR, Komeya AY, Kirkcaldy RD, et al. Cluster of Neisseria gonorrhoeae Isolates With High-level Azithromycin Resistance and Decreased Ceftriaxone Susceptibility, Hawaii, 2016. Clin Infect Dis 2017;65(6):91823. doi: $10.1093 / \mathrm{cid} / \mathrm{cix} 485$

69. Ross JD, Harding J, Duley L, et al. LB1.5 The efficacy and safety of gentamicin for the treatment of genital, pharyngeal and rectal gonorrhoea: a 
randomised controlled trial. Sexually Transmitted Infections

2017;93(Suppl 2):A42-A43. doi: 10.1136/sextrans-2017-053264.106

70. Handsfield HH, Dalu ZA, Martin DH, et al. Multicenter trial of single-dose azithromycin vs. ceftriaxone in the treatment of uncomplicated gonorrhea. Azithromycin Gonorrhea Study Group. Sex Transm Dis 1994;21(2):107-11.

71. Zheng S, Matzneller P, Zeitlinger M, et al. Development of a population pharmacokinetic model characterizing the tissue distribution of azithromycin in healthy subjects. Antimicrob Agents Chemother 2014;58(11):6675-84. doi: 10.1128/AAC.02904-14

72. Matzneller P, Krasniqi S, Kinzig M, et al. Blood, tissue, and intracellular concentrations of azithromycin during and after end of therapy. Antimicrob Agents Chemother 2013;57(4):1736-42. doi: 10.1128/AAC.02011-12

73. Crokaert F, Hubloux A, Cauchie P. A Phase I Determination of Azithromycin in Plasma during a 6-Week Period in Normal Volunteers after a Standard Dose of 500mg Once Daily for 3 Days. Clin Drug Investig 1998;16(2):1616.

74. Buono SA, Watson TD, Borenstein LA, et al. Stemming the tide of drugresistant Neisseria gonorrhoeae: the need for an individualized approach to treatment. J Antimicrob Chemother 2015;70(2):374-81. doi: 10.1093/jac/dku396 [published Online First: 2014/10/22]

75. European Medicines Agency. Review of Quinolone- and fluoroquinolonecontaining medicinal products.

https://www.ema.europa.eu/en/medicines/human/referrals/quinolonefluoroquinolone-containing-medicinal-products2018 [accessed 10th January 2019.

76. Sathia L, Ellis B, Phillip S, et al. Pharyngeal gonorrhoea - is dual therapy the way forward? Int J STD AIDS 2007;18(9):647-8. doi: 10.1258/095646207781568556

77. Barbee LA, Kerani RP, Dombrowski JC, et al. A retrospective comparative study of 2-drug oral and intramuscular cephalosporin treatment regimens for pharyngeal gonorrhea. Clin Infect Dis 2013;56(11):1539-45. doi: $10.1093 / \mathrm{cid} / \mathrm{cit} 084$

78. Ison CA, Hussey J, Sankar KN, et al. Gonorrhoea treatment failures to cefixime and azithromycin in England, 2010. Euro Surveill 2011;16(14)

79. Unemo M, Golparian D, Syversen G, et al. Two cases of verified clinical failures using internationally recommended first-line cefixime for gonorrhoea treatment, Norway, 2010. Euro Surveill 2010;15(47)

80. Pichichero ME, Casey JR. Safe use of selected cephalosporins in penicillinallergic patients: a meta-analysis. Otolaryngol Head Neck Surg 2007;136(3):340-7. doi: 10.1016/j.otohns.2006.10.007

81. Zagursky RJ, Pichichero ME. Cross-reactivity in beta-Lactam Allergy. The journal of allergy and clinical immunology In practice 2018;6(1):72-81.e1. doi: 10.1016/j.jaip.2017.08.027 [published Online First: 2017/10/12]

82. Kirkcaldy RD, Weinstock HS, Moore PC, et al. The efficacy and safety of gentamicin plus azithromycin and gemifloxacin plus azithromycin as treatment of uncomplicated gonorrhea. Clin Infect Dis 2014;59(8):108391. doi: 10.1093/cid/ciu521 
83. TAPSALL JW, SHULTZ TR, LIMNIOS EA, et al. Failure of Azithromycin Therapy in Gonorrhea and Discorrelation With Laboratory Test Parameters. Sexually Transmitted Diseases 1998;25(10):505-08.

84. Haimovici R, Roussel TJ. Treatment of gonococcal conjunctivitis with singledose intramuscular ceftriaxone. American journal of ophthalmology 1989;107(5):511-4. [published Online First: 1989/05/15]

85. Brocklehurst P. Antibiotics for gonorrhoea in pregnancy. Cochrane Database Syst Rev 2002(2):CD000098. doi: 10.1002/14651858.CD000098

86. Cavenee MR, Farris JR, Spalding TR, et al. Treatment of gonorrhea in pregnancy. Obstet Gynecol 1993;81(1):33-8.

87. Ramus RM, Sheffield JS, Mayfield JA, et al. A randomized trial that compared oral cefixime and intramuscular ceftriaxone for the treatment of gonorrhea in pregnancy. Am J Obstet Gynecol 2001;185(3):629-32. doi: 10.1067/mob.2001.117662

88. FitzGerald M, Thirlby D, Bell G, et al. National standards for contact tracing in gonorrhoea. Royal College of Physicians, National Audit Development Programme in Sexual Health. Int J STD AIDS 1996;7(4):301. doi: 10.1258/0956462961917870

89. McClean H, Radcliffe K, Sullivan A, et al. 2012 BASHH statement on partner notification for sexually transmissible infections. International Journal of STD \& AIDS 2013;24(4):253-61. doi: 10.1177/0956462412472804

90. Mensforth $S$, Radcliffe K. Is it time to reconsider epidemiological treatment for gonorrhoea? Int J STD AIDS 2018;29(10):1043-44. doi: $10.1177 / 0956462418787611$

91. Jephcott AE. Microbiological diagnosis of gonorrhoea. Genitourin Med 1997;73(4):245-52.

92. Wind CM, Schim van der Loeff MF, Unemo M, et al. Test of Cure for Anogenital Gonorrhoea Using Modern RNA-Based and DNA-Based Nucleic Acid Amplification Tests: A Prospective Cohort Study. Clin Infect Dis 2016;62(11):1348-55. doi: 10.1093/cid/ciw141 